\title{
The Hakka Yong Tau Foo: A Typical Symbol and Identity of Hakka Food Cultural Value in Food-Anthropological Perspective
}

\author{
Yang Liao, Shaodi He \\ School of Public Administration, South China Agricultural University, Guangzhou, China \\ Email: liaoyang410@126.com
}

How to cite this paper: Liao, Y. and He, S.D. (2018) The Hakka Yong Tau Foo: A Typical Symbol and Identity of Hakka Food Cultural Value in Food-anthropological Perspective. Open Journal of Social Sciences, 6, 1-13.

https://doi.org/10.4236/jss.2018.62001

Received: December 22, 2017

Accepted: January 28, 2018

Published: January 31, 2018

Copyright $\odot 2018$ by authors and Scientific Research Publishing Inc. This work is licensed under the Creative Commons Attribution International License (CC BY 4.0). http://creativecommons.org/licenses/by/4.0/

\begin{abstract}
There is wealth of researches of Hakka food in the origin of Chinese cultural food, the Hakka food diversification, regional needs, Hakka sense, etc. to discuss how and why Hakka Yong Tau Foo affected the lives of Hakka people from historiography and Hakka cultural views. Based on these large number of Hakka literature researches, this article interprets how the Hakka Yong Tau Foo becomes a typical symbol and Hakka cultural identity as basic essential factors of Hakka food culture and Hakka detective value. Thus, the Hakka Yong Tau Foo has become important component in Hakka Food cultural development, and also has drawn the attention of people to Hakka Food. From the Food-anthropological perspective, we could see how Hakka Yong Tau Foo communicates with other food culture in the context of globalization.
\end{abstract}

\section{Keywords}

Hakka Yong Tau Foo, Typical Symbol, Hakka Cultural Identity, Hakka Food Culture

\section{Introduction}

For decades, Hakkaology has gradually developed into an integrated subject which synchronized the history, anthropology, ethnology, folklore, linguistics and so on [1]. As a background of this study, there are five statements about the Hakka sub-ethnic. Luo Xianglin, a representative of Hakkaology, thought the Hakka is a product of multiple culture of the Han people migrating to south China and south local ethnic minorities. The Hakka keeps the Chinese advanced Central plains culture [2]. The second statement had published by Fang Xuejia, who thought that Hakka people is the commonwealth that the Han people who 
migrated to south China and ancient Bei Yue people who is the subject of Hakka that they live together [3]. The third statement is the culture identity according to Xie Chong Guang. Hakka is a cultural concept that proved Hakka is the integration of indigenous people who came from Gan (Jiangxi), Min (Fujian) and Canton (Guangdong) and North Han Chinese who migrated south China [4]. The fourth category is the ethnic identity. Referring to Sow-Theng Leong, Hakka is an ethnic group which plays a role in the emergence of sense of Hakka [5]. The last one is dialect group of Hakka. Wang Dong thought Hakka is a dialect group in area Gan Nan (south of Jiangxi), Min-Si (western Fujian) and Eastern Guangdong from time to time in history [6]. Based on these statements of Hakka, the research of Hakka Yong Tau Foo is primarily located in the Hakka region of Eastern Guangdong.

There are few articles about the Hakka Yong Tau Foo, but previous studies have explored nomenclature and origin of Hakka Yong Tau Foo and the complex of Hakka hometown. Two papers of the Hakka Yong Tau Foo have played a role in the research of Hakka Yong Tau Foo. One is in Zhou Yufang's paper, the Origin of Bean Curd with Fillings in Hakka Cuisine, which expressed Niang To$\mathrm{fu}$ is a typical dish combing the traditional Central Plains dietary custom with the mode of production and life style of destination area of migrants in the south based on naming Niang Fotu (Yong Tau Foo) that lies in ancient Chinese traditional cooking method [7]. The other one is On the Foundation of Hakka Culture from the Origin of Stuffed Bean Curd, which was found that the origin of Hakka Yong Tau Foo had Chinese cultural roots such as long history, Hakka tradition and psychology to connect the primitive culture [8]. However, there are few discussions on the symbol and cultural identities of Hakka Yong Tau Foo in the numerous articles and researches of Hakka food culture. In this article, we discuss how the Hakka Yong Tau Food interprets a typical symbol and becomes a cultural identity of Hakka food to analyze the dietetic value of Hakka food culture from the perspective of Food anthropology in the social change. On one hand, could the Hakka Yong Tau Foo be a typical symbol of Hakka Food culture to communicate with other dietetic culture in the globalization? On the other hand, in the dietetic diversity era, could the Hakka Yong Tau Foo be given the cultural identity in the process of consumption? It is thus evident that could help people broaden their horizon to understand the mean of Hakka Yong Tau Foo by perspective and approach of Hakka food culture. Therefore, we can see that how Hakka Yong Tau Foo plays an important role in Hakka food culture to communicate with other food culture.

\section{The Hakka Yong Tau Foo as a Typical Symbol of Hakka}

In this paper, Hakka Yong Tau Foo is character transcription of Hakka regional dialectal accent, which means the bean curd with fillings in Hakka cuisine. In Zhou Yufang's paper, she translated the bean curd with fillings in Hakka Cuisine into Chinese mandarin, called niang tofu. She discussed the practical signific- 
ance of Hakka food culture which is based on the naming to Hakka niang tofu sourcing of history culture of Hakka bean curd [7]. Actually, this paper is not discussed the name of this Hakka cuisine or what do we call it, we explored that has it been a typical symbol and how it could be? Therefore, we called Hakka Yong Tau Foo as Hakka regional dialectal accent. Based on the paper, On the Foundation of Hakka Culture from the Origin of Stuffed Bean Curd, a history culture of Hakka Yong Tau Foo was dug into the ancient literature, books, folks which is the "The Origin of stuffed tofu" so deeply that it proved that the stuffed bean curd in the $19^{\text {th }}$ century has appeared in Hualounty and Nanjing county with its origin of the Ming Dynasty [8]. We can see that it has been a long diffusion process since the Hakka Yong Tau Foo emerging into the public. According to $\mathrm{Qu}$ Ming-An (2007) [9], the fundamental characteristics of symbols include those of community, subject, multi meaning, space-time and continuance. Also, food may serve as: 1) an indicator of social relation such as marriage, gift, feast and banquet; 2) a symbol of caste, class, hierarchy and status; and 3) a metaphor thought which the mechanism of self-construction with regard to ethnicity and identity can be discerned (Goody 1982; Mintz 1985; Ohnuki-Tierney 1993; Sahlins 1976) [10].

First of all, in reference to Li Bing (2009) [11], Yong Tau Foo is an evolution of dumplings which is full of the feeling of missing homeland and ancestor. Hakka ancestor was inspired by some characteristics of central china's food culture to create a Hakka Yong Tau Foo. Therefore, the Hakka Yong Tau Foo has two characteristics of a symbol, one is time-space coherence, the other historical inheritance. According to Zhong LinBing (2007) [12], The Hakka Yong Tau Foo and The North Making Dumplings, there is a close relative between Hakka Yong Tau Foo and Dumplings in Chinese traditional diet, production process and the relationship of both configuration and value. There are many folk narratives to talk about the origin of the Hakka Yong Tau Foo, which come from the dumplings. Based on participant observation, we find the Hakka Yong Tau Foo is a formable dumpling in shape, production and sentiment as shown in Figure 1.

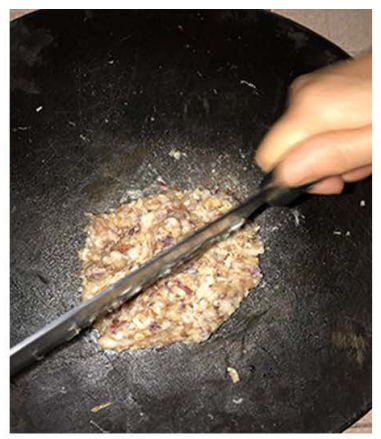

Making mince

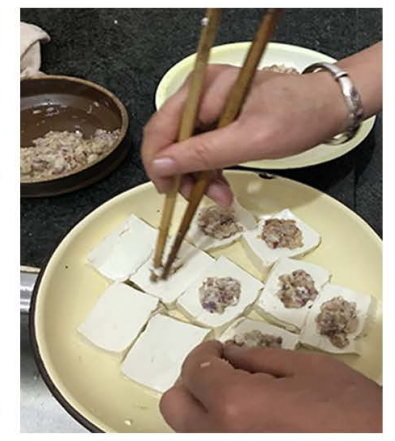

Stuffing mince into tofu

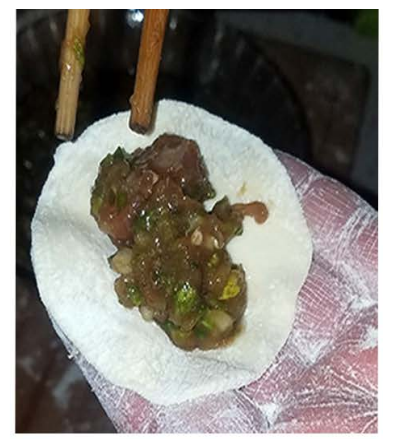

Stuffing mince into dumpling skin

Figure 1. The comparison between the Hakka Yong Tau Foo and dumpling. 
What is mean by a formable dumpling? In appearance of white color, there are facial similarities that Tofu and dumpling skin are the same as white. In a part of food ingredient, there are internal similarities between the Hakka Yong Tau Foo and dumplings for a part of food materials, such as meat stuffing. In the making process, Hakka Yong Tau Foo and dumplings are both need to stuff minced meat, the former is open that people can see the minced meat, the latter is close that people cannot see the minced meat.

The appearance of Hakka Yong Tau Foo as a typical symbol is a long process which is the Hakka people who need to constantly deal with the dietetic differences of cultural identity in the Hakka migration. Adapting diets is one of the best way to regain a Hakka cultural sense of symbol and identity for Hakka ancestor who could acclimatize themselves properly to environment. All in all, in the form the Hakka Yong Tau Foo is so similar to the Chinese Northern dumplings that Hakka Yong Tau Foo are coveted as a formable dumpling, even the symbol of memory and connect with time and space. According to Luo shunfen (2008) [13], the Hakka cleverly use the similar food production methods with the local ingredients to fulfill their feelings of homesick, and then find an emotional affinity of considering abroad as home. The Hakka Yong Tau Foo is the innovation of dumplings in order to have a connection with the feeling of hometown, to regain the sense of superiority of strong cultural identity for Hakka. Hakka Yong Tau Foo is about creating exemplary food, in which the initial dumpling is still recognizable, but which establish Hakka Yong Tau Foo through the evolution of form and shape as shown in Figure 2.

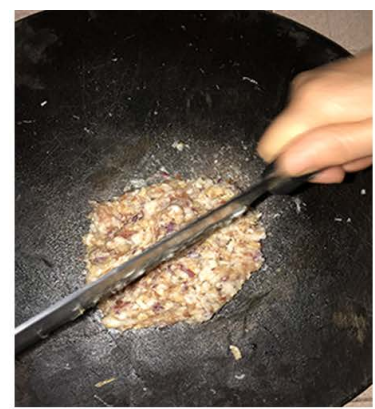

Making mince

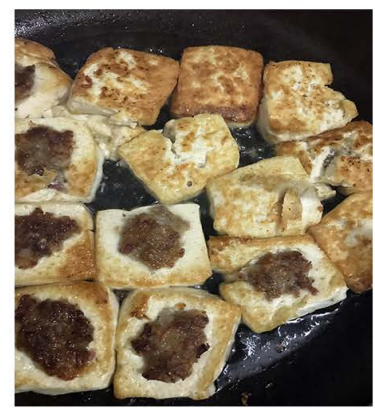

The Raw Hakka Yong Tou Fu
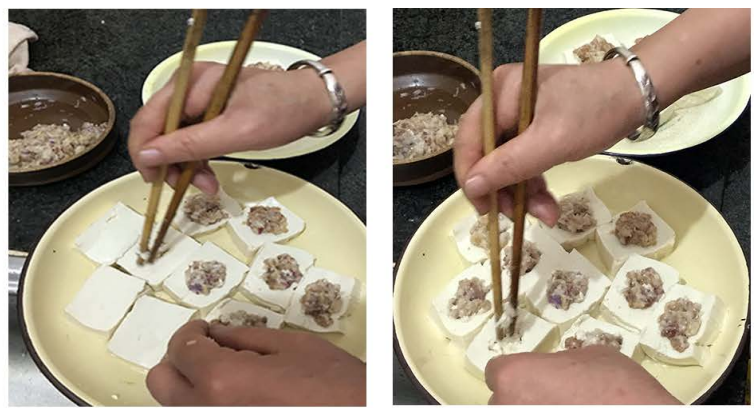

Stuffing tofu

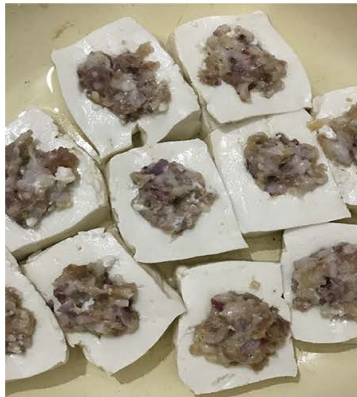

Cooking the Hakka Yong Tou Fu

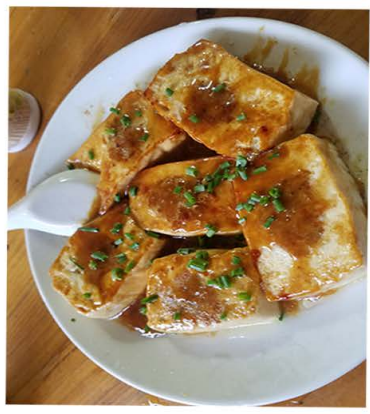

The Hakka Yong Tou Fu

Figure 2. The procedure of the Hakka Yong Tau Foo and dumpling. 
What is more, from the functional perspective of food anthropology, Hakka Yong Tau Foo is not only for edibility, but also a kind of special activity to strengthen the cohesion of the Hakka family. Making tofu is one of most important part in the traditional process of Hakka Yong Tau Foo. The production process of making tofu is no less than the complex process of making dumpling skin. Tofu is the skin of Hakka Yong Tau Foo. Yan Xiuhong, Huang Chunbin \& He Xiaokang (2014) [14], Making tofu is step one for making Hakka Yong Tau Foo. The steps of making tofu are from picking beans, breaking beans, soaking beans, grinding soy milk, pouring soy milk in a, filtering bean dregs, boiled soy milk, jelling soy milk, spreading the gauze of tofu, wrapping tofu, squeezing tofu and so on, then the tofu takes shape. Each of Hakka family has had to specialize and focus on one steps of making tofu and Hakka Yong Tau Foo. It is a family recreational activity that the Hakka family shared out the work of making food and cooperate with the family members. As Fei Xiantong's point of view (2012) [15], relaxation from muscular and nervous stress after hard work is a physiological necessity. In so fat as recreation needs collective action, social institutions develop for this function. The collective action in recreation reinforce the social ties among the participant the social ties among the participant. Thus, its function goes beyond pure physiological relaxation. Making Hakka Yong Tau Foo as a recreation and collective action need to mobilize the members of family to participate in making Hakka Yong Tau Foo. This collective action creates a feeling of harmony to encourage more open communication and strengthening the sense of cohesion for Hakka family. Making Hakka Yong Tau Foo became a celebration and symbol of Hakka family meal. As for Hakka ancestor, the process of making Hakka Yong Tau is that they enjoy. This dietary collective action delighted Hakka ancestor who displaced by the war or other reasons in new geographic location. So, the dietary function of Hakka Yong Tau Foo extends it with recreational function. Hakka Yong Tau Foo has become a typical symbol of Hakka food to strengthen family cohesion.

"Today, in Hakka restaurant, the Hakka Yong Tau Foo as a Hakka Food consumption habit is indispensable food. The Hakka Yong Tau Foo is a custom for the Hakka food. If there are no the Hakka Yong Tau Foo in the Hakka restaurants or Hakka Banquet, the Hakka will not recognize that is the perfect banquet. Because Tau Foo in Hakka dialect is a homophone for 'wealth'." some Hakka people said according to interviews of participant observation as shown in Figure 3. We can learn from the community, subjectivity and multi meaning of Hakka that what the factors or features of Hakka Yong Tau Foo as a typical symbol of Hakka are.

The Hakka Yong Tau Foo which breaks through the limitations of time and space has bridged that gap, to connect Hakka posterity and Hakka ancestor about how Hakka wish Hakka could be, with what they are really like in the Hakka social change and globalization. Hakka Yong Tau Foo and dumplings are from the same original cultural consciousness to express the tolerance and flexibility of Chinese culture. It is continuance between the dumplings and the 


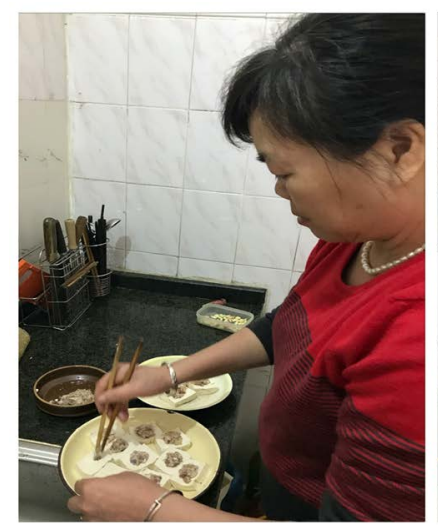

A Hakkka Women making Hakka Yong Tau Foo

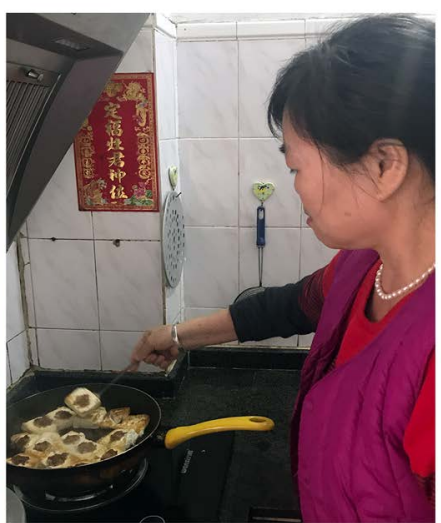

A Hakka Woman cooking Hakka Yong Tau Foo

Figure 3. An interview to a Hakka Woman of participant observation.

Hakka Yong Tau Foo. Both of them rooted deeply in the humanistic value and geography living, but they show their own features in their own historical inheritance, community, subjectivity, multiplicity, space-time coherence and continuance. Ages of interchange among different food culture of ethnic groups have involved the Chinese food culture which created food material wealth and spiritual property for the future generations. Hakka make the Hakka Yong Tau Foo as a typical symbol of the values that are linked to the central China's food culture that can help them to teach their children and grandchildren to identify with taste and the identity of the food culture. In the process of migration and settling, making Hakka Yong Tau Foo is a realistic expression of homesickness because the Central China's food culture is the foundation of the Hakka food culture. If we see the dumpling as the symbol as the Central China's food, we can say that the Hakka Yong Tau Foo is a new symbol of Hakka food which was created by Hakka in the new hometown in the support of realistic expression of Hakka adjustment. The Hakka Yong Tau Foo as typical symbol gradually became a link between the Hakka group food culture and the Central China's food culture in the processes of nature and social change. Making and eating Hakka Yong Tau Foo as a family recreation and collective action is effective medium to comprehend the multiple combination relations between a manifestation and target object of Hakka Yong Tau Foo in a particular space-time context. Hakka Yong Tau Foo is a sign that shows the adaptation of Hakka eating habits. From the perspective of food anthropology, Hakka Yong Tau Foo become a typical symbol of Hakka Foo as a kind of spiritual consolation and transcendence, which is sublimated into a part of Hakka food culture.

\section{The Hakka Yong Tau Foo as a Cultural Identity of Hakka}

From the food anthropological perspective, food has the function to express their own cultural identity. Based on the typical symbol, Hakka Yong Tau Foo evolved into a meaning of cultural identity for Hakka. According to Sidney W. 
Mintz (1986) [16], what is behind the meaning of food is the history, power relations, cultural preferences and classification systems with complex rights. In reference to Sidney Cheung (2008) [17], Cultural anthropologists have regarded food as an important medium to convey ideas and concepts. So, why did Hakka evolve Hakka Yong Tau Foo a cultural identity?

According to Stuart Hall (1990) [18], Hall theorizes two ways of reflecting on "cultural identity": first, identity understood as a collective, shared history among individuals affiliated by race or ethnicity that is considered to be fixed or stable; and second, identity understood as unstable, metamorphic, and even contradictory- an identity marked by multiple points of similarities as well as differences. Identity is not spoken of, never complete, always in process, and always constitutes within, not outside, representation. Similarly, Hakka Yong Tau Foo has a cultural identity of Hakka Foo that is always in process, and always constitute within Hakka culture. On the one hand, Hakka Yong Tau Foo as one of Hakka eating habits is Hakka's an emotional connection to the past to express Hakka subjectivity and collectivity. Hakka food culture and central Chinese traditional culture can be traced back to the same root and have deep original foundations. Hakka Yong Tau Foo as a cultural identity of Hakka food is a kind of recognition of Chinese traditional culture. On the other hand, Hakka Yong Tau Foo as a Hakka cultural identity is a medium to communicate with other food culture. This medium is not only an expression of historical inheritance and collective emotion for Hakka to convey their yearning for their hometown and recall their ancestors in social change and globalization, but also a subliminal symbol and an ego emotion that has been shared with ancestors and posterity. It is best way of cultural identity is the taste of hometown in the global cultural interflow. Hakka Yong Tau Foo reconstructs Hakka identity of being a Hakka for their posterity in the historical process of globalization. Hakka use their Hakka food as a body memory of taste to adapt to the new life, environment, social change and cultural integration. Making Hakka Yong Tau Foo is creating Hakka food, story and history.

In today's globalized world, Hakka Yong Tau Foo is a Hakka dietetic behavior of cultural self-consciousness to keep its own identity and feature and develop cultural exchanges during the cultural melting pot of globalization. This Hakka dietetic behavior of cultural self-consciousness has created an emotional expression of cultural root-seeking by reflection of traditional eating habits to live in the current life. Wu Xue li (2011) [19], has mentioned that, "the pursuit of cultural identity is often directed towards the future cultural trend through modern reflection on the long history of traditional culture." As for Hakka, creating a food like ancestor's food is a good way of communication to gain a position in the global food culture. The fundamental reason why Hakka Yong Tau Foo is endowed with cultural identity is that Hakka need to get a consolation of taste to constructing the balance between the individual and Hakka identity, the individual existence and the balance of the home and country in the world. 


\section{The Hakka Yong Tau Foo as a Part of Hakka Food Culture}

Tan Zhiguo (2004) [20], points out that: "only when human beings meet the basic needs such as food, clothing, housing etc. that they will create some new and secondary, derivable feature surroundings. The surrounding is called 'culture"'. Zhao Rongguang and Xie Dingyuang (2000) [21], has noted that making explorations into the food cultural cannot continue without the processing techniques of foodstuff, the production of food, the science, technology and art of dietary consumption, and the custom, tradition, thought and philosophy of diet basic components. The Hakka dietetic cultural value of Hakka food culture when we discussed is based on the Yong Tau Foo as a typical symbol and cultural identity. We think the Hakka Foo cultural value of Hakka Yong Tau Foo was mainly represented in two aspects: one is Hakka dietetic natural selection of biotechnology for body need; other is cultural selection of psychologic for Hakka spiritual need.

What are the reasons that the Hakka Yong Tau Foo is the natural selection of biotechnology for Hakka?

First of all, Tofu, the technology of soybean, an ancient food laid foundation for Hakka to make Hakka Yong Tau Fu. William A. Havilland, Harald E.L. et al. (2014) [22], thought that "organisms mush has a regular supply of food, as well as a reliable way to get and use it." One of most important part of Hakka Yong Tau Foo is the Tofu and the method of making tofu. The soybeans are the raw material of tofu. The raw material of tofu- soybean has an indispensable role and status in the farming civilization of China for thousands of years. When Chinese ancestors did not know the specific nutritional composition of soybean, the soybean as a raw material of tofu has been one of favorite food that is deeply loved by Chinese people. Today, we know that why the tofu is a significant food for Chinese ancestors with technological advancements. In the research of Sun Ming Jun and Ding An Lin (1999) [23], they found that soybean is the nutritional origination of vegetable proteins and fats which contains not only much more protein and fat, but also many valuable biological active materials including soybean lipoxygenase, trypsin inhibitor, isoflavone saponin, phosphatides oligosaccharides etc. As A Bite of China, a hugely popular TV documentary mentioned, the invention of tofu, however, completely changed the fate of soybeans. The disadvantages of soybeans were eliminated by reason or unconsciously, as the ancient Chinese transformed soybeans into tofu. The value of soybean protein to human body reached a climax with the invention of tofu. The Hakka Yong Tau is also a wisdom as the ancient Chinese Hakka transformed soybeans into Hakka Yong Tau Foo. Hakka are showing their adaptability through the Hakka Yong Tau. And thus, tofu has been sublimated. Tofu and Hakka Yong Tau Foo resembled each other in spirit and came down in one continuous lien in dietetic nutrition, dumpling and Hakka Yong Tau Foo has a derivative aesthetic feel. Hakka Yong Tau Fu provides the body needs of nutrition, such as, natural ingredients: Soy protein. 
Moreover, Hakka Yong Tau Foo is a constraint on geographic factor and local harvest. As we know, the Hakka moved and settled in after a series of migrations. In the context of social change, the Hakka improved the recipe of tofu. These migrations had prompted them to change the ancestors eating habits. Li Bing (2009) [11], discussed that Hakka Food culture is a new dietetic culture which is created by the Han people of the Central Plains after settling down in the south, and combining the customs and habits of the local people with the customs and habits of the local people in the south according to the climatic conditions in the South and the customs and habits of the local people. Hakka Yong Tau Foo is a living embodiment of this new dietetic culture, a Hakka Food culture. Zhou Yu Fang (2012) [7], thought that according to the specific geography, the core environment, Hakka created a rich regional cuisine through the continuous improvement of innovation and formed a unique eating custom of local to inherit the cooking skill from the ancestors of the Chinese Central plains. Thus, the Hakka Yong Tau Foo is created by the Hakka with the Chinese traditional cooking skill and the specific food customs of the migratory geographical environment.

The final point above further emphasizes that the Hakka Yong Tau Foo played a role of high quality protein supply of body need for Hakka who were living in the era of scarcity. The greatest thing of Hakka Yong Tau Foo is the meat stuffing. "Stuffing" is feature of Hakka food that can increase the mouth feel and flavor of the meat. In Wang (2013) [24] has stated that most of Hakka settled in hard-to-reach mountain villages during the past, the poverty had forced them to seek a balance between cooking skill and delicious and of high quality to nutrients of body need. The meat stuffing plays a prominent part in the Tofu, a cheap and high quality, which shows that the meat of Hakka Yong Tau Foo is precious and delicious. Those hard and necessitous time in Hakka place, meat is an unheard-of luxury for many Hakka, Hakka Yong Tau Foo as a typical Hakka Foo provided Hakka with sufficient protein for body need. In particular, tofu can help attain a flavor balance when being cooked with greasy fatty meat.

We can see a harmonious state between the food needs of the Hakka and the geographical environment through the Hakka Yong Tau Foo, one of the particularly identified with Hakka food culture. Zhang Ying-bin (2010) [8] has noted that Hakka Yong Tau Foo was not the aesthetic design at first, but the economic consideration in the mountain area. People assume that Hakka in remote places are friendly and hospitable. Hakka would try their best to entertain their relatives and friends and make these guests eat and drink better based on their local nature, social custom and economic condition, even if they are poor. The dietetic cultural value of Hakka is rooted in the nature and suited local circumstance to work hard, as classic Chinese philosophy of Dao, a harmony and unity between human and nature.

For another cultural selection, Hakka Yong Tau Foo is an emotional need of Hakka spiritual need. How does Hakka Yong Tau Foo of Hakka Food Culture 
reflect the cultural selection of an emotional need of Hakka?

Above all, the dietetic cultural value of Hakka Yong Tau Fu shows a part of Hakka humanity, history, the food consumption process of dietetic science, cooking technology and art. William A. Havilland, Harald E.L. et al. (2014) [22], the unique way of human adaptation is its ability to produce and reproduce culture, which enables us to creatively adapt to a series of environments. As a formable dumpling, Hakka Yong Tau Foo, is filled with the art of behavior through its functions which are not only esculent function, but also recreational function for Hakka family. Hakka Yong Tau Foo's meaning of dietetic consumption refers to the consumption of material information in the diet that meets the needs of production or life. As a symbol of Hakka food culture, Hakka Yong Tau Foo plays an important role of Hakka food language to communicate with other food culture to express the complex of Hakka homeland, Hakka family cohesion and Hakka dietetic consumption habits. What's more, Hakka elders like junior to learn the spirit of Hakka ancestor through this Hakka dish. It is a gift of taste memories from one generation.

Furthermore, it is worth nothing that Hakka Yong Tau Foo contains the Hakka food cultural values based on the ideology and philosophy of the traditional Chinese humanism. Hakka is an important clan and distinctive Han Chinese subgroup. A common food custom and culture is a link to maintain an ethnic group because the ethnic culture, to a certain extent, determines the development trend of ethnic group. Tofu is a symbol of virtue which is contented in poverty and devoted to things spiritual in Chinese conventional moral notion and Confucianism. Hakka follow this thought and use those cultural connections to strengthen the invisible ties that hold humanity together, and to deepen their understanding of change as making the best of each situation with a flexible way. When the Hakka had settled down, they felt an intense yearning for the peaceful life. Hakka food culture is an adjustment reaction of Hakka identity which is invisible mental factor. The central philosophy in traditional Chinese cultures has actually shaped not only Hakka food culture but Hakka eating habits. Hakka Yong Tau Foo place minced meat stuffing with rich and heavy in the tofu with a thin and light taste together, just like the Hakka culture connected traditional Chinese food custom, more experiences into Hakka life in the social changes, Therefore, the creating of Hakka Yong Tau Foo Tofu is not only a cultural retainment and transmission, but also one of the most recognized in the local and a global collection action.

Last, Hakka Yong Tau Foo is the "host" sign of Hakka table as a treat. To maintain relevancy in today's society of food multi-cultural communication, Hakka find a position of Hakka food which is based on food cultural exchange to develop the Hakka food consumption in the context of globalization. Chen $\mathrm{Su}$ Hua (2006) [25], mentioned that eating habits are formed in a social environment People live, which means the traditional and the custom environment through learning consciously, the customs and traditions deeply rooted in 
People's heart and created ethic culture human being in their environment. As for Hakka, they realize the origins, formation, characteristics and development of food culture in social changes. Therefore, through this meal, it can be showed that deep in Hakka's mind, they know clearly where they come from and where they want to go. They still maintain an innocence and purpose in their heart. With those, even away from home, they will try really hard on their own to earn their lives, nourish their well-beings and obtain cultural identity in this big melting pot.

The dietetic cultural value of the Hakka Yong Tau Fu helps Hakka to establish their position and create the Hakka dietetic custom/habit in the global dietetic consumption. Hakka Yong Tau Fu has always been the one on Hakka's table for feast or banquet, no matter when and where, or how rich or poor they are. The dietetic cultural value of Hakka Yong Tau Fu is related to both natural and cultural selection, which are coexistence to mutually indicate the value of Hakka Yong Tau Fu.

\section{Conclusion}

Hakka Yong Tau Fu as a typical symbol and Hakka Food cultural identity shows a part of dietetic cultural value of Hakka humanity, history, dietetic science, cooking technology and art. The dietetic cultural value of Hakka Yong Tau Fu is based on conforming to both nature and human which is the spirit of traditional Chinese culture. Tasting Hakka food tries to understand the Hakka food culture and knows more about the Hakka culture. Due to the complex of Hakka migrants in an increasingly globalized society, we have no sufficient funding, time and manpower to seek development of different kinds, sense of Hakka and changes of Hakka Yong Tau Foo in Southeast Asia. This paper is not only to seek the cultural meaning of Hakka food through the Hakka Yong Tau Foo as a typical symbol and Hakka food cultural identity at the micro level in food-anthropology perspective, but also interest people to understand the characteristics of Hakka food as a part of Hakka routine in changing society. In particular, it is an innovative extension of Hakkaology which focuses on the foodways with Hakka food cultural values.

\section{References}

[1] Zhou, D.M. (2015) The Ecology, Ethic Group and Hakka Culture in Border Region of Jiangxi, Min and Guangdong During the Ming and Qing Dynasties. China Social Sciences Press, Beijing, 1-2.

[2] Luo, X.L. (1975) An Introduction to Hakka Research. Guting shuwu, Taipei, 27-28.

[3] Fang, X.J. (1994) The Origin of Hakka. Guangdong Higher Education Press, Guangzhou, 16-17.

[4] Xie, C.G. (1995) A New Exploration of The Origin of Hakka. Fujian Education Press, Fuzhou, 52-53.

[5] Leong, S.-T. (1997) Migration and Ethnicity in Chinese History: Hakkas, Pengmin, and Their Neighbors. Stanford University Press, Stanford, 63-64. 
[6] Wang, D. (2007) Mountain, Water and People: The New Phrase of Hakka Origin. East China Normal University Press, Shanghai, 31-32.

[7] Zhou, Y.F. (2012) The Origin of Bean Curd with Fillings in Hakka Cuisine. Journal of Wuyi University (Social Sciences Edition), 1, 27-29, 94. http://dx.chinadoi.cn/10.3969/j.issn.1009-1513.2012.01.007

[8] Zhang, Y.-B. (2010) On the Foundation of Hakka Culture from the Origin of Stuffed Bean Curd. Journal of Jia Ying University (Philosophy Social Sciences), 28, 5-11. http://doi:10.3969/j.issn.1006-642X.2010.10.001

[9] Qu, M.A. (2007) On Fundamental Characteristics of Symbols. Ethno-National Studies, 5, 56-65. https://doi.org/10.3969/j.issn.0256-1891.2007.05.006

[10] Cheung, S. and Wu, D.Y.H. (2001) Hakka Restaurants: A Study of the Consumption of Food in Post-war Hong Kong Society. In: Wu, D.Y.H. and Tan, C.B., Eds., Changing Chinese Foodways in Asia, The Chinese University Press, Hong Kong, 81-95.

[11] Li, B. (2009) On the Impact of Geographical Environment upon Hakka Food Culture. Journal of Jiaying University, 3, 113-115. https://doi.org/10.3969/j.issn.1006-642X.2009.03.028

[12] Zhong, L.B. (2007) The Hakka Making Toufu and Northern China's Making Jiaozi. Root Exploration, 5, 22-24. https://doi.org/10.3969/j.issn.1005-5258.2007.05.006

[13] Luo, S.F. (2008) The Hakka Dietary Homeland Complex. Jiangxi Food Industry, 3, 10-13. https://doi.org/10.3969/j.issn.1674-2435.2008.03.003

[14] Yan, X.H., Huang, C.B. and He, X.K. (2014) The Tufo in the Hakka life. Hakka Cultural Heritage Vision, 1, 12-15.

[15] Fei, H.T. (2010) Peasant Life in China-A Field Study of Country Life in the Yangtze Valley. Foreign Language Teaching and Research Press, Beijing, 111-113.

[16] Mintz, S.W. (1985) Sweetness and Power: The Place of Sugar in Modern History. Viking Penguin, New York, 126-127.

[17] Cheung, S. (2008) Food Anthropology, Anthropology. China Renmin University Press, Beijing, 240-254.

[18] Hall, S. (1990) Cultural Identity and Diaspora. Blackwell Publishing, Hoboken, NJ, 232-235.

[19] Wu, X.L. (2011) On "Cultural Root Seeking” and Identity in the Context of Globalization. Lanzhou Academic Journal, 9, 194-198. https://doi.org/10.3969/j.issn.1005-3492.2011.09.038

[20] Tan, Z.G. (2004) Research on Chinese Dietary Culture from the Perspective of Cultural Anthropology. Journal of Hubei University of Economics, 1, 124-127. https://doi.org/10.3969/j.issn.1672-626X.2004.02.026

[21] Zhao, R.G. and Xie, D.Y. (2000) An Introduction to the Diet Culture. China Light Industry Press, Beijing, 17-27.

[22] Havilland, W.A., Prins, H.E.L., Mcbride, B. and Walrath, D. (2014) Cultural Anthropology: The Human Challenge. $13^{\text {th }}$ Edition, China Machine Press, Beijing, 153-155.

[23] Sun, J.M. and Ding, A.L. (1999) The Role of Soybean Processing Production in the Food Industry. Science and Technology of Food Industry, S1, 166-169. https://doi.org/10.13386/j.issn1002-0306.1999.s1.046

[24] Wang, Q.J. (2013) The Hakka Stuffing Food and The Hakka sentiment. Hakka Cultural Heritage Vision, 1, 13-15. 
[25] Chen, S.H. (2006) A Discussion on the Character of Dietary Culture and Its Discipline Position. Culinary Science Journal of Yangzhou University, 4, 6-10.

https://doi.org/10.3969/j.issn.1009-4717.2006.04.003 